\title{
LMTK3 knockdown retards cell growth and invasion and promotes apoptosis in thyroid cancer
}

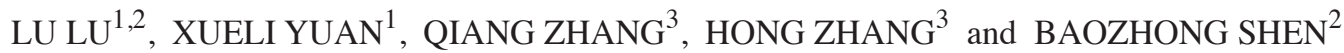 \\ ${ }^{1}$ Department of Medical Oncology, The Fourth Affiliated Hospital of Harbin Medical University; \\ ${ }^{2}$ Molecular Imaging Center, Harbin Medical University, Harbin, Heilongjiang 150001; \\ ${ }^{3}$ Center for Stem Cell and Translational Medicine, School of Life Sciences, Anhui University, Hefei, Anhui 230601, P.R. China
}

Received November 30, 2015; Accepted November 25, 2016

DOI: $10.3892 / \mathrm{mmr} .2017 .6262$

\begin{abstract}
Lemur tyrosine kinase-3 (LMTK3) is a member of the serine/threonine tyrosine kinase family, which is thought to be involved in tumor progression and prognosis. The purpose of the present study was to determine the diagnostic significance and therapeutic targets in thyroid cancer. ELISA assay was used to detect the protein expression of serum LMTK3. Immunohistochemistry and reverse transcription-quantitative polymerase chain reaction were employed to measure the expression of LMTK3. Flow cytometry was used to determine the cell cycle. Transwell assay was used to measure the invasion and migration of SW579 cells and terminal deoxynucleotidyl transferase dUTP nick end labeling (TUNEL) assay to detect cell apoptosis. The LMTK3 level was positively associated with disease stage and pathological type, whereas there was no correlation between LMTK3 level and gender, age, tumor size or lymph node metastasis. The serum LMTK3 level was significantly increased in 102 thyroid carcinoma patients compared with 52 benign thyroid tumor patients and 50 healthy volunteers $(\mathrm{P}=0.001)$. The protein and mRNA expression of LMTK3 was markedly higher in thyroid cancer patients compared with patients with benign thyroid tumors. Notably, LMTK3 knockdown retarded proliferation, invasion and migration in SW579 cells. In addition, downregulation of LMTK3 promoted apoptosis in SW579 cells. These findings indicated that LMTK3 knockdown retards the growth of thyroid cancer cells partly through inhibiting proliferation, invasion, migration and inducing apoptosis in SW579 cells. It may serve as a useful diagnostic biomarker and a novel therapeutic target for patients with thyroid cancer.
\end{abstract}

Correspondence to: Professor Baozhong Shen, Molecular Imaging Center, Harbin Medical University, 37 Yiyuan Street, Nangang, Harbin, Heilongjiang 150001, P.R. China

E-mail: sbzxslwyx@163.com

Key words: lemur tyrosine kinase-3, thyroid cancer, proliferation, apoptosis

\section{Introduction}

Thyroid cancer is one of the more common endocrine malignancies with a rapidly rising incidence in recent years (1-3). Histologically it consists mainly of anaplastic thyroid cancer (ATC), papillary thyroid cancer (PTC), and follicular thyroid cancer (FTC) (4). Almost all thyroid cancers are derived from follicular cells that comprise the simple unicellular epithelium of normal thyroid. Follicular thyroid cell-derived tumours include PTC and FTC, poorly differentiated thyroid cancer (PDTC) and undifferentiated ATC, whereas parafollicular C cell-derived medullary thyroid cancer accounts only for a small proportion (2 to 3\%) of cases (5). PTC and FTC are classified as differentiated thyroid cancers (DTCs) which may be cured with surgery or radioiodine treatment. The 10 -year survival rate for PTC and FTC is $>90 \%$, while PDTC and undifferentiated ATC have poor prognoses (6). Approximately 5 to $23 \%$ of DTC patients develop distant metastases, which are the main cause of mortality (7). The appropriate extent of surgery for thyroid cancer is controversial: Certain researchers recommend partial, and others total, thyroidectomy; others advocate prophylactic central cervical lymph node dissection, while only rarely do researchers suggest lymphadenectomy (8-10). Although radioactive iodine is effective, the appropriate use and dosage remain controversial $(11,12)$. Recently, molecular analysis of thyroid cancer has been usually applied for diagnostic purposes, involving preoperative fine-needle biopsy specimens, as well as to define targetable pathways altered in the disease to guide the clinical trials of drug therapy $(13,14)$.

Hormones promote cell proliferation and augment random genomic mutation, thus increasing the opportunity for tumorigenicity (15). In fact, there is an obvious gender disparity in the occurrence of DTC, with females having three times the incidence of DTC than males $(16,17)$. Previously, researchers have demonstrated that thyroid cancer cells express higher levels of estrogen receptors (ERs) and progesterone receptors (PRs) compared with normal cells (18). Two ERs, ER $\alpha$ and $\mathrm{ER} \beta$, have different biological functions (19). In thyroid cancer cells, ER $\alpha$ expression is enhanced; however, ER $\beta$ expression is low or absent (11). An ER $\alpha$ agonist has been revealed to enhance the proliferation of thyroid cancer cells, while an ER $\beta$ agonist did not (16,20). Therefore, an ER $\alpha$-mediated signalling pathway may be critical for the proliferation of thyroid cells. 
The lemur tyrosine kinases (LMTKs) belong to a family of transmembrane serine/threonine tyrosine kinases, and have been shown to be localized in cytoplasmic membrane vesicles and involved in endosomal trafficking (21). Accumulating evidence indicates an important role for LMTK3 in various types of cancer. RNA interference screening has identified LMTK3 as a potential therapeutic target in colon cancer and leukemia cells $(22,23)$. In breast cancer, LMTK3 isoform knockdown repressed ER $\alpha$ activity, while an LMTK1/2 isoform knockdown did not. Additionally, LMTK3 was identified to interact with $\mathrm{ER} \alpha$ in vivo and phosphorylation of $\mathrm{ER} \alpha$ by LMTK3 was revealed to protect ER $\alpha$ from proteosomal degradation (24).

Similarly to other cancers, thyroid cancer initiation and progression is mediated through the accumulation of multiple genetic and epigenetic alterations of critical molecules and signalling pathways (25). Identification of the altered molecular makers is crucial for the diagnosis and treatment of thyroid cancer. LMTK3 has been recognized as a potential biomarker or a prognostic marker for various malignancies, including breast cancer, gastric cancer and colorectal cancer (26-28). However, the clinical significance of LMTK3 and its association with thyroid cancer has yet to be identified. In the present study, LMTK3 expression in thyroid cancer was examined and its associated clinical significance was explored.

\section{Materials and methods}

Cell culture. The human thyroid carcinoma cell line SW579 was purchased from the American Type Culture Collection (American Type Culture Collection, Manassas, VA, USA). SW579 was cultured in RPMI-1640 (Gibco Invitrogen; Thermo Fisher Scientific, Inc., Waltham, MA, USA) with $10 \%$ fetal bovine serum (FBS; HyClone ${ }^{\mathrm{TM}}$, Logan, UT, USA). Cells were kept at $37^{\circ} \mathrm{C}$ in a humidified incubator containing $5 \%$ $\mathrm{CO}_{2}$.

Patients and serum. The serum specimens were obtained from patients at the Fourth Hospital of Harbin Medical University (Harbin, Heilongjiang, China) who had not undergone surgery. All serum specimens were derived from 106 thyroid carcinoma patients (26 male and 80 female; age range: 25 to 72 years; average age: $48.26 \pm 14.67$ years) and 52 benign thyroid tumor patients. Patients who had undergone any form of pre-operative chemotherapy and/or radiation therapy were excluded. None of the patients enrolled in this study suffered from any other type of cancer. The clinical and pathological features are presented in Table I. A total of 52 benign thyroid tumor patients and 50 healthy volunteers were enrolled. A serum separator tube was used to isolate serum. Blood samples were allowed to clot for $2 \mathrm{~h}$ at room temperature before centrifugation for $15 \mathrm{~min}$ at $1,000 \mathrm{x}$. Thereafter, serum was collected and immediately placed at $-80^{\circ} \mathrm{C}$ to avoid protein or mRNA degradation. All procedures were approved by the ethics committee of the Fourth Hospital of Harbin Medical University (Heilongjiang Province, China).

ELISA assay for LMTK3. The level of LMTK3 was measured using a human LMTK3 ELISA kit (MyBioSource, Inc., San Diego, CA, USA) according to the manufacturer's protocol. Briefly, whole blood samples $(100 \mu \mathrm{l})$ were added to high-binding polystyrene plates coated with capture monoclonal antibody for LMTK3. Immobilized antigen was detected with diluted biotinylated secondary antibody (dilution, 1:100), followed by horseradish peroxidase-conjugated streptavidin. For calibration, recombinant LMTK3 protein and two control standards were performed in parallel with the tested samples on each plate.

Immunohistochemistry. Formalin-fixed, paraffin-embedded tissue sections $4 \mu \mathrm{m}$ thick were chosen for immunohistochemical staining. Anti-LMTK3 human monoclonal antibody was purchased from Abcam (Cambridge, UK; cat. no. ab137260; dilution, 1:1,000). The tissue sections were dewaxed in xylene and then hydrated in a series of graded alcohols. Specimens were heated in $10 \mathrm{mM}$ sodium citrate buffer $(\mathrm{pH} \mathrm{6.0)}$ and subsequently EDTA (pH 8.0), prepared for LMTK3, at $100^{\circ} \mathrm{C}$ for $5 \mathrm{~min}$ to expose the antigens. The specimens were then washed with PBS ( $\mathrm{pH}$ 7.4) and incubated with $3 \% \mathrm{H}_{2} \mathrm{O}_{2}$ at $37^{\circ} \mathrm{C}$ for $15 \mathrm{~min}$ to eliminate endogenous peroxidase activity, and $5 \%$ bovine serum albumin for $30 \mathrm{~min}$ to reduce non-specific binding. The slides were kept overnight at $4^{\circ} \mathrm{C}$ with primary antibodies (LMTK3 antibody with a dilution of 1:200). Following washing, the specimens were incubated with peroxidase-labeled polymer conjugated to goat anti-human LMTK3 (dilution, 1:4,000; cat. no. A0201; Beyotime Institute of Biotechnology, Haimen, China) in Tris-HCl buffer at room temperature for $30 \mathrm{~min}$. Signals were visualized with diaminobenzidine and the slides were counterstained with hematoxylin. For negative controls, the primary antibody was substituted with PBS.

Reverse transcription-quantitative polymerase chain reaction $(R T-q P C R)$. First, the cells were incubated with antibiotic-free medium for $24 \mathrm{~h}$ prior to transfection. For the LMTK3 knockdown, the cells were transfected with siRNA against LMTK3 using Lipofectamine 2000 (Invitrogen: Thermo Fisher Scientific, Inc.). Transfection complexes were added to the medium at final oligonucleotide concentration of $50 \mathrm{nM}$. Total RNA samples from human tissues were isolated using Trizol reagent (Invitrogen; Thermo Fisher Scientific, Inc.) according to manufacturer's protocol. Total RNA $(1 \mu \mathrm{g})$ was reverse-transcribed using High-Capacity cDNA Reverse Transcription kit (Applied Biosystems; Thermo Fisher Scientific, Inc.) to obtain complementary DNA (cDNA). The SYBR Green PCR Master Mix kit (Applied Biosystems; Thermo Fisher Scientific, Inc.) was applied in RT-qPCR to quantify the level of LMTK3, with GAPDH as an internal control. The RT-qPCR was performed on a 7500 FAST Real-Time PCR System (Applied Biosystems; Thermo Fisher Scientific, Inc.) for 40 cycles. The primers were designed as follows, The primers for GAPDH: Sense primer: 5'-AAGAAGGTGGTGAAGCAGGC-3', antisense primer: 5'-TCCACCACCCAGTTGCTGTA-3'. The primers for LMTK3: sense primer: 5'- TCGGCTTCAAGGAATTTG AGA-3', antisense primer: 5'-GGGTGGTCATGTCTGAGT GTGA-3'.

Small interfering RNA (siRNA) and transfection. The target sequence GGAAUUUGAGAACCCUGAATT, for mouse LMTK3 was purchased from Shanghai GenePharma Co., Ltd. 
(Suzhou, China). As a control, LMTK3 siRNA negative control (NC) was also used: UUCUCCGAACGUGUCACGUTT. The cells were transfected with siRNAs using Lipofectamine reagent (Invitrogen; Thermo Fisher Scientific, Inc.) according to the manufacturer's protocol and kept for a further $48 \mathrm{~h}$ prior to being used in the subsequent experiments.

Cell proliferation assay. SW579 cells were seeded into 96-well plates and treated with saline, LMTK3 siRNA or NC. The serum-free medium was removed and the cells were cultured with regular culture medium for a further $48 \mathrm{~h}$. To monitor cell survival, SW579 cells were incubated for $4 \mathrm{~h}$ with $0.5 \mathrm{mg} / \mathrm{ml}$ MTT (Sigma-Aldrich; Merck Millipore, Darmstadt, Germany), and dissolved in $150 \mu$ l dimethylsulfoxide (DMSO; Sigma-Aldrich; Merck Millipore). Absorbance was recorded at $490 \mathrm{~nm}$ using an Easy Reader 340 AT (SLT Labinstruments $\mathrm{GmbH}$, Crailsheim, Germany). Results are presented as the percentage of survival, taking the control as $100 \%$ survival. Experiments were repeated six times.

Terminal dUTP nick end labeling (TUNEL) assay. Apoptotic SW579 cells in different groups were detected using a TUNEL assay as previously described (29). Air-dried slides were fixed with $4 \%$ paraformaldehyde for $30 \mathrm{~min}$ at room temperature, washed three times with PBS, and then permeabilized with $1 \%$ Triton X-100 for $4 \mathrm{~min}$ at $4^{\circ} \mathrm{C}$. Subsequently, each slide was removed to a terminal deoxynucleotidyl transferase (TdT) -labeled nucleotide mix and kept at $37^{\circ} \mathrm{C}$ for $60 \mathrm{~min}$ in the dark. Slides were rinsed twice with PBS and then counterstained with $10 \mathrm{mg} / \mathrm{ml} \mathrm{4,6-diamidino-2-phenylindole} \mathrm{for} 5 \mathrm{~min}$ at $37^{\circ} \mathrm{C}$.

Cell cycle analysis. The effect of LMTK3 siRNA on cell cycle distribution was measured by flow cytometric analysis of the DNA content of cell nuclei following staining with propidium iodide (PI; Sigma-Aldrich; Merck Millipore). SW579 cells were seeded into 60-mm flasks, and allowed to attach overnight. The cells were rinsed with PBS and fixed in $75 \%$ ethanol overnight at $4^{\circ} \mathrm{C}$. The cells were then treated with $80 \mathrm{mg} / \mathrm{ml}$ RNaseA (Sigma-Aldrich; Merck Millipore) and $50 \mathrm{mg} / \mathrm{ml}$ PI for $30 \mathrm{~min}$, and analyzed using a Coulter Epics XL Flow Cytometer (Beckman Coulter, Miami, FL, USA).

Cell migration and invasion assays. Transwell chambers with a pore size of $8 \mathrm{~mm}$ (Corning Costar, Inc., Corning, NY, USA) were used for cell migration and invasion assay. Cells were brought to $60 \%$ confluency and transfected with the siRNA for $48 \mathrm{~h}$. For migration assays, cells were digested with $0.25 \%$ trypsin (Beyotime Institute of Biotechnology), resuspended in serum-free medium and placed in the upper chamber. As a chemoattractant, the lower chamber contained $10 \%$ FBS. Cells were cultured at $37^{\circ} \mathrm{C}$ in $5 \% \mathrm{CO}_{2}$ for $24 \mathrm{~h}$, and non-migrating cells were removed with a cotton swab. Migrated cells were washed twice with PBS, fixed in 100\% methanol and stained with hematoxylin. Stained cells were viewed under a microscope (magnification, x200), and the number of migrated cells was counted in five random fields. For invasion assays, the upper chamber was precoated with Matrigel mixed with serum-free medium (diluted at 1:3; BD Biosciences, San Jose, CA, USA). Following solidification of the mixture, $5 \times 10^{5}$ cells in serum-free medium were placed into the upper chamber. The

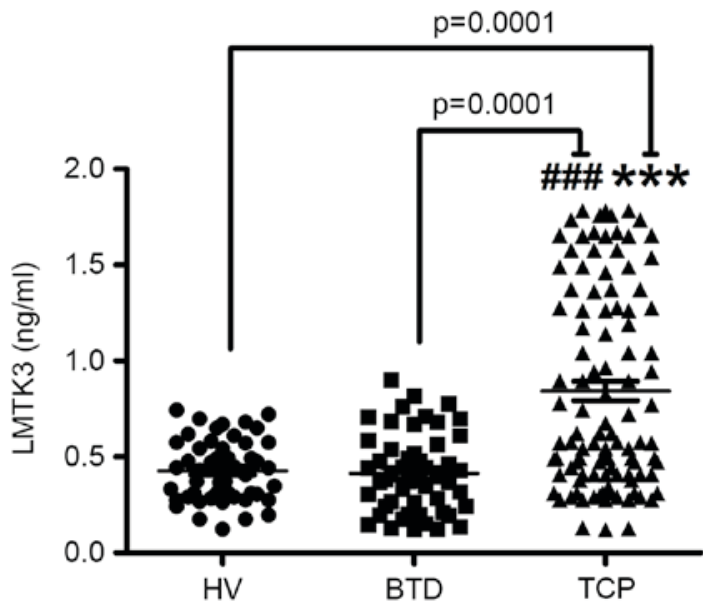

Figure 1. Serum level of LMTK3 measured by ELISA in TCP, BTD and HV Markedly elevated serum LMTK3 levels were detected in TCP compared with BTD and HV $(n=6)$. Data are expressed as the mean \pm standard error of the mean. ${ }^{* * *} \mathrm{P}<0.001$ vs. HV; ${ }^{\# \#} \mathrm{P}<0.001$ vs. BTD. LMTK3, lemur tyrosine kinase-3; TCP, thyroid cancer patients; BTD, benign tumor diseases; HV, healthy volunteers.

lower chamber contained $10 \%$ FBS as a chemoattractant. Cells were cultivated at $37^{\circ} \mathrm{C}$ in a humidified incubator containing $5 \% \mathrm{CO}_{2}$ for $24 \mathrm{~h}$, and non-invading cells were removed with a cotton swab. Invasive cells were fixed, stained and counted. Stained cells were viewed under a microscope (magnification, $\mathrm{x} 200$ ), and the number of migrated cells was counted in five random fields. Assays were performed in three independent experiments.

Statistical analysis. All quantitative data are expressed as the mean \pm standard error of the mean and analysed using SPSS software, version 13.0 (SPSS Inc., Chicago, IL, USA). Two-tailed unpaired Student's $t$-test and one-way analysis of variance were used for statistical evaluation of the data. $\mathrm{P}<0.05$ was considered to indicate a statistically significant difference.

\section{Results}

Histopathological characteristics of thyroid cancer. Histological diagnoses and tumor features were derived from 106 thyroid cancer patients ( 52 with benign tumors and 54 with malignant tumors) and 50 healthy volunteers (Table I). In the whole group (thyroid cancer patients only, $\mathrm{n}=106$ ), the mean tumor size was $9.5 \pm 0.9 \mathrm{~mm}$ and the average age was $48.67 \pm 2.26$ years. Male and female subgroup features were compared.

Serum LMTK3 level was markedly increased in thyroid cancer patients. It is known that LMTK3 could become a potential therapeutic target in multiple tumors. Therefore, an ELISA assay was performed to confirm whether LMTK3 was involved in the pathological process in thyroid cancer. Serum LMTK3 was derived from 106 thyroid cancer patients, 52 benign thyroid tumor diseases and 50 healthy volunteers, respectively. As presented in Fig. 1, serum expression of LMTK3 was markedly elevated in patients with thyroid cancer $(0.68 \pm 0.10 \mathrm{ng} / \mathrm{ml})$ compared with those with benign thyroid tumors $(0.38 \pm 0.06 \mathrm{ng} / \mathrm{ml}, \mathrm{t}=5.708)$ and in the normal tissues 
Table I. Clinical and histopathological characteristics in patients with thyroid cancer.

\begin{tabular}{|c|c|c|c|c|}
\hline Characteristic & Whole $(\mathrm{n}=106)$ & Male $(n=26)$ & Female $(\mathrm{n}=80)$ & $\begin{array}{c}\text { Male vs. female } \\
\text { (P-value) }\end{array}$ \\
\hline Age (years) & $48.67 \pm 2.26$ & & & $0.019^{\mathrm{a}}$ \\
\hline$\geq 50$ & 37 (34.9) & $14(53.8)$ & $23(28.8)$ & \\
\hline$<50$ & $69(65.1)$ & $12(46.2)$ & $57(71.2)$ & \\
\hline Tumor size (mean \pm SEM) & $9.5 \pm 0.9$ & $10.2 \pm 0.6$ & $9.1 \pm 1.3$ & 0.465 \\
\hline Degree of differentiation & $4 / 34 / 64$ & $1 / 8 / 15$ & $3 / 26 / 49$ & 0.997 \\
\hline (poor/intermediate/good) & $(3.9 / 33.3 / 62.7 \%)$ & $(4.2 / 33.3 / 62.5 \%)$ & $(3.8 / 33.3 / 62.8 \%)$ & \\
\hline Capsule invasion & $62(58.5 \%)$ & $13(0.5 \%)$ & $49(61.5 \%)$ & 0.315 \\
\hline Vascular invasion & $26(24.5 \%)$ & $3(2.9 \%)$ & $21(26.9 \%)$ & 0.076 \\
\hline Stages & & & & 0.727 \\
\hline $\mathrm{I}+\mathrm{II}$ & $89(83.9 \%)$ & $22(84.6 \%)$ & $67(83.8 \%)$ & \\
\hline III+IV & $17(16.1 \%)$ & $4(15.4 \%)$ & $13(16.2 \%)$ & \\
\hline Pathological types & & & & 0.141 \\
\hline Papillary cancer & $68(64.1 \%)$ & $17(65.4 \%)$ & $51(63.8 \%)$ & \\
\hline Follicular cancer & $23(21.7 \%)$ & $6(23 \%)$ & $17(21.2 \%)$ & \\
\hline Medullary cancer & $12(11.3 \%)$ & $3(11.5 \%)$ & $9(11.3 \%)$ & \\
\hline Undifferentiated Cancer & $3(2.8 \%)$ & $2(7.6 \%)$ & $1(1.3 \%)$ & \\
\hline
\end{tabular}

${ }^{a} \mathrm{P}<0.05$, comparison between the ages of male subgroup and female subgroup. SEM, standard error of the mean.

of healthy volunteers $(0.41 \pm 0.09 \mathrm{ng} / \mathrm{ml}, \mathrm{t}=5.5304)$. The results further indicated that the LMTK3 level was closely associated with the aggressive stages of thyroid cancer, whereas no correlations were identified between gender, age, tumor size and lymph node metastasis, as presented in Table II $(\mathrm{P}=0.48$, $\mathrm{P}=0.389, \mathrm{P}=0.643$ and $\mathrm{P}=0.752$ for gender, age, tumor size and lymph node metastasis, respectively). In addition, significant differences in the four pathological types of serum LMTK3 level were identified (Table II).

LMTK3 expression in patients with thyroid cancer. To further ascertain the involvement of LMTK3 in the development of thyroid cancer, immunohistochemistry, RT-qPCR and western blotting were employed to measure the expression of LMTK3 in thyroid cancer and benign thyroid tumor tissues. Immunohistochemistry results indicated a stronger expression of LMTK3 in tissue sections from human thyroid cancer samples, and LMTK3 was expressed not only in the cell nuclei, but also in the cytoplasm of tumor cells. By contrast, LMTK3 staining was negative in corresponding benign thyroid tumor tissues (Fig. 2A). The results demonstrated that the mRNA level for LMTK3 was almost 4-fold higher than that of the corresponding benign thyroid tumor disease tissues (Fig. 2B). Additionally, western blotting results identified that LMTK3 in human thyroid cancer samples was markedly higher compared with benign thyroid tumor tissues (Fig. 2C).

LMTK3 knockdown inhibited cell cycle and retarded proliferation. Flow cytometric analysis and MTT assays were employed to validate the role of LMTK3 in regulating the cell cycle and proliferation in SW579 cells. As presented in Fig. 3A, the mRNA level of LMTK3 was substantially decreased by treatment with LMTK3 siRNA for $48 \mathrm{~h}$ compared with the negative control (NC). Consistently with Fig. 3A, western blot analysis demonstrated that the protein expression of LMTK3 was also clearly reduced in the LMTK3 siRNA-treated group (Fig. 3B). The effect of LMTK3 knockdown on cell cycle distribution was determined to gain insights into the mechanism of its anti-proliferative activity. As illustrated in Fig. 3C, LMTK 3 siRNA treatment for $48 \mathrm{~h}$ resulted in an accumulation of cells in the G2/M phase that was accompanied by a reduction in cells with G0/G1 DNA content. Previously, decreased LMTK3 activity has been demonstrated in breast cancer, where it is considered to inhibit cell proliferation (17). Therefore, an MTT assay was performed to further document the effect of LMTK3 knockdown on SW579 cell proliferation. The results identified that LMTK3 knockdown clearly inhibited cell proliferation (Fig. 3D), and indicated that LMTK3 silencing is an effective inhibitor of SW579 cell growth.

LMTK3 knockdown suppresses the migration and invasion of SW579 cells. As presented in Fig. 4A, the migratory capability of SW579 cells transfected with the LMTK3 siRNA was clearly reduced compared with the control group $(\mathrm{Ctrl})$. However, the cells had approximately similar migration abilities in the NC and the Ctrl groups. To further determine whether LMTK3 knockdown contributes to mitigate the SW579 cells invasion, an invasion assay was performed by using 24-well Boyden chambers coated with Matrigel. As presented in Fig. 4A, the number of SW579 cells was clearly fewer than that observed for the NC and Ctrl groups. These data strongly evidently that downregulation of LMTK3 could mediate a reduction in the migration and invasion of SW579 cells (Fig. 4B).

LMTK3 knockdown promoted SW579 cells apoptosis. To evaluate the extent of apoptosis in SW579 cells, apoptotic 
Table II. LMTK3 protein levels and clinical features in thyroid cancer.

\begin{tabular}{|c|c|c|c|c|}
\hline Clinical pathological feature & No. & Mean \pm SEM & t-value & P-value \\
\hline Gender & & & -0.713 & 0.48 \\
\hline Male & 26 & $0.68 \pm 0.06$ & & \\
\hline Female & 80 & $0.62 \pm 0.01$ & & \\
\hline Age (years) & & & -0.866 & 0.389 \\
\hline$\geq 50$ & 37 & $0.67 \pm 0.05$ & & \\
\hline$<50$ & 69 & $0.61 \pm 0.03$ & & \\
\hline Tumour size & & & -0.465 & 0.643 \\
\hline$<2 \mathrm{~cm}$ & 54 & $0.61 \pm 0.04$ & & \\
\hline$\geq 2 \mathrm{~cm}$ & 52 & $0.65 \pm 0.02$ & & \\
\hline Lymph node metastasis & & & -0.318 & 0.752 \\
\hline Negative & 78 & $0.62 \pm 0.48$ & & \\
\hline Positive & 28 & $0.65 \pm 0.35$ & & \\
\hline Stage & & & -4.805 & 0.0001 \\
\hline $\mathrm{I}+\mathrm{II}$ & 89 & $0.54 \pm 0.09$ & & \\
\hline III+IV & 17 & $0.95 \pm 0.10$ & & \\
\hline Pathological types & & & & 0.0001 \\
\hline Papillary cancer & 68 & $0.44 \pm 0.01$ & & \\
\hline Follicular cancer & 23 & $0.96 \pm 0.06$ & & \\
\hline Medullary cancer & 12 & $0.86 \pm 0.03$ & & \\
\hline Undifferentiated cancer & 3 & $1.17 \pm 0.01$ & & \\
\hline
\end{tabular}

SEM, standard error of the mean.

A

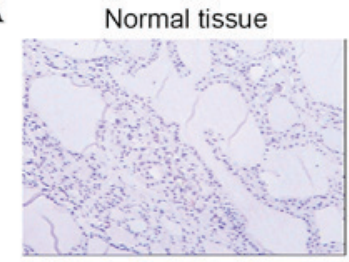

Follicular cancer

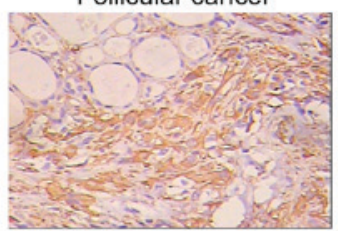

B
Benign thyroid diseases

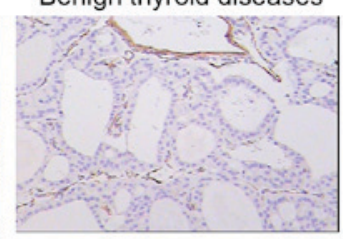

Medullary cancer

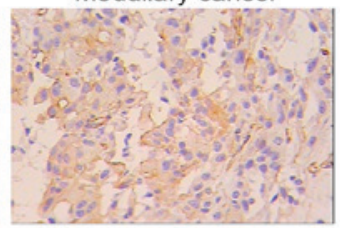

C

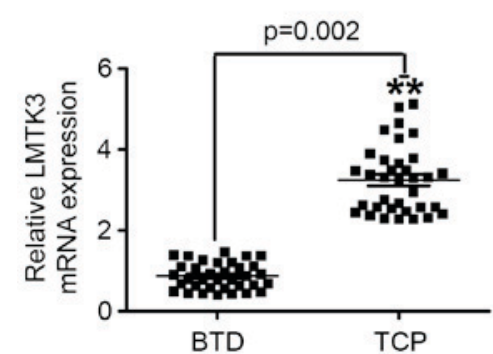
$151 \mathrm{kDa}$

Papillary cancer

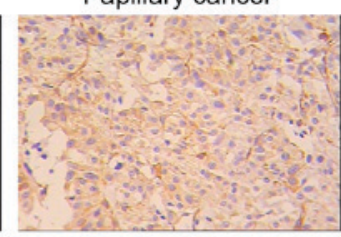

Undifferentiated cancer

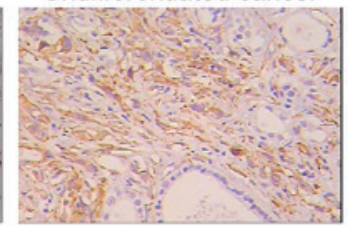

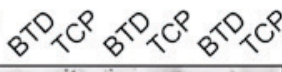
$36 \mathrm{kDa}$

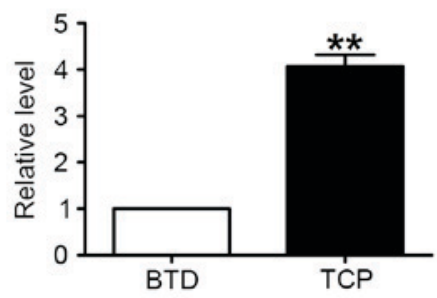

Figure 2. LMTK3 level measured in TCP, BTD and HV tissue. (A) LMTK3 expression visualized by immunohistochemistry staining (original magnification, x400; scale bar $=100 \mu \mathrm{m}$ ). (B) The mRNA expression of LMTK3 was markedly elevated in TCP compared with BTD tissue samples. (C) Protein levels of LMTK3 were increased in TCP compared with BTD tissue samples. Data are expressed as the mean \pm standard error of the mean $(n=35)$. ${ }^{* *} \mathrm{P}<0.01$ vs. BTD group. LMTK3, lemur tyrosine kinase-3; GAPDH, glyceraldehyde 3-phosphate dehydrogenase; TCP, thyroid cancer patients; BTD, benign tumor diseases; $\mathrm{HV}$, healthy volunteers. 
A

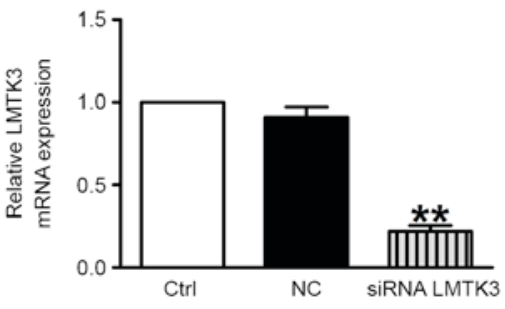

$\mathrm{C}$

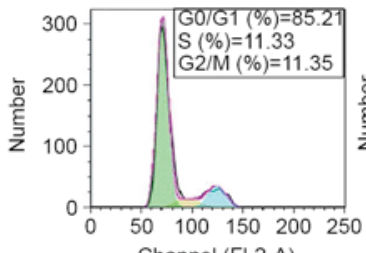

Channel (FL2-A)

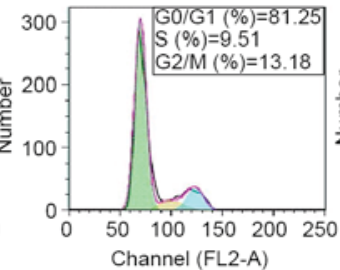

Channel (FL2-A)

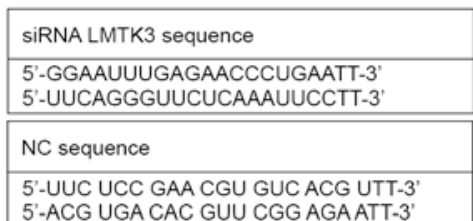

5'-ACG UGA CAC GUU CGG AGA ATT-3'

\begin{tabular}{|llll|}
\hline Groups & G0/G1 phase & S phase & G2/M phase \\
\hline Control & $85.03 \pm 3.12$ & $10.63 \pm 2.21$ & $11.27 \pm 1.67$ \\
\hline NC & $82.17 \pm 3.37$ & $10.26 \pm 3.17$ & $12.03 \pm 1.87$ \\
\hline LMTK3 siRNA & $65.28 \pm 4.72^{*}$ & $13.38 \pm 2.46$ & $20.21 \pm 2.43^{*}$ \\
\hline
\end{tabular}

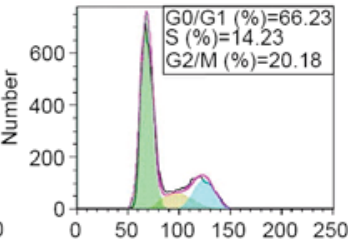

Channel (FL2-A)
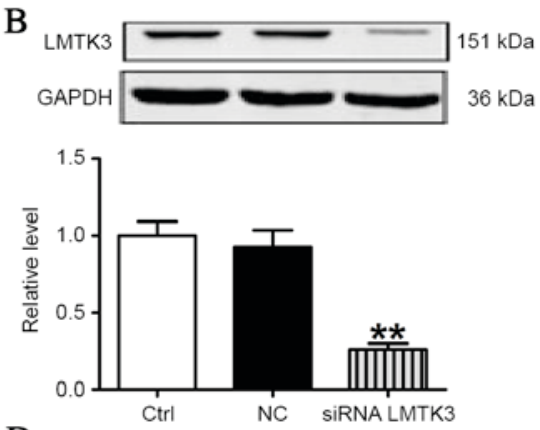

D

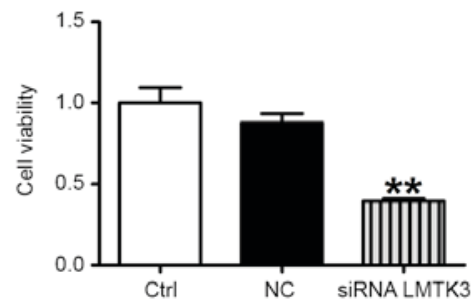

Figure 3. LMTK3 knockdown retarded cell cycle and inhibited proliferation of SW579 cells. (A) The mRNA level of LMTK3 was reduced in the siRNA LMTK3 treated group compared with NC $(n=6)$. (B) Protein expression of LMTK3 was significantly inhibited by treatment with LMTK3 siRNA compared with NC ( $\mathrm{n}=3)$. (C) The effect of LMTK3 knockdown on cell cycle distribution in SW579 cells. Histograms illustrate SW579 cells treated with LMTK3 siRNA or exposed to NC, respectively (n=6). (D) Cell proliferation assessed by using an MTT assay. The result demonstrated that cell proliferation was decreased by treatment with LMTK3 siRNA, $\mathrm{n}=6$. Data are expressed as the mean \pm standard error of the mean. ${ }^{* *} \mathrm{P}<0.01 \mathrm{vs}$. NC. LMTK3, lemur tyrosine kinase-3; GAPDH, glyceraldehyde 3-phosphate dehydrogenase; siRNA, small interfering RNA; NC, negative control.

A
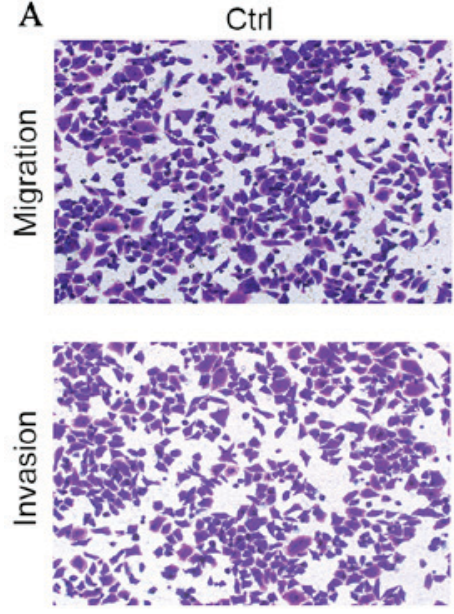

B

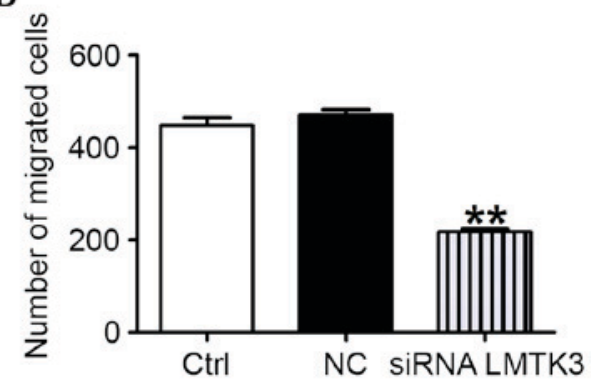

NC
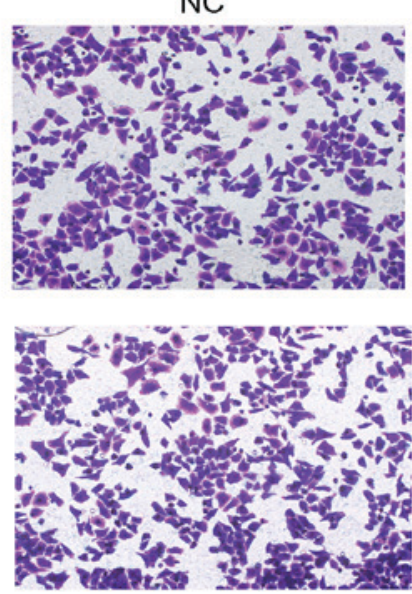

SiRNA LMTK3
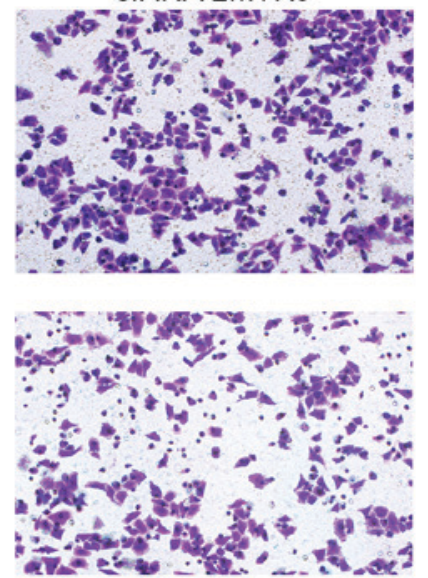

$\mathrm{C}$

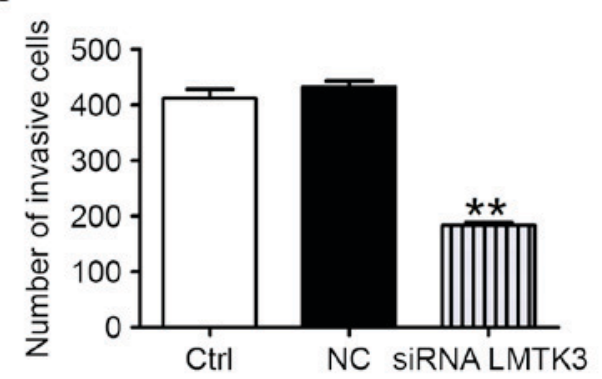

Figure 4. LMTK3 siRNA inhibits the migration and invasion of SW579 cells. (A) Migration/invasive cells were imaged following staining with hematoxylin (original magnification, x200). (B) Histogram of the number of migrated cells. (C) Histogram of the number of invaded cells. Data are expressed as the mean \pm standard error of the mean $(n=6) ;{ }^{* *} \mathrm{P}<0.01$ vs. NC. Ctrl, control group; $\mathrm{NC}$, negative control; siRNA, small interfering RNA; LMTK3, lemur tyrosine kinase-3. 
A

A
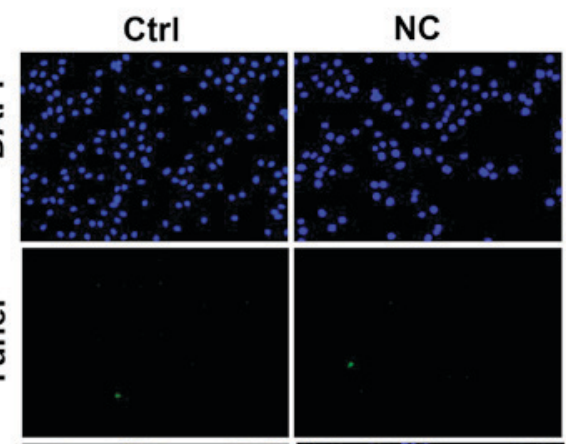

SIRNA LMTK3
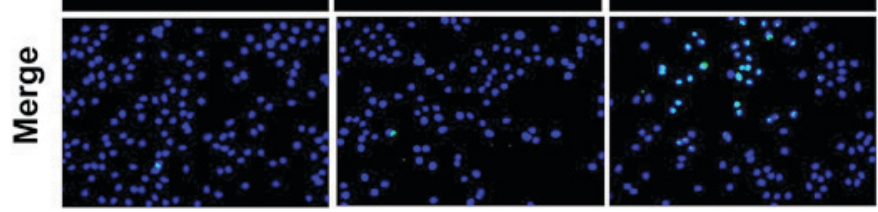

B

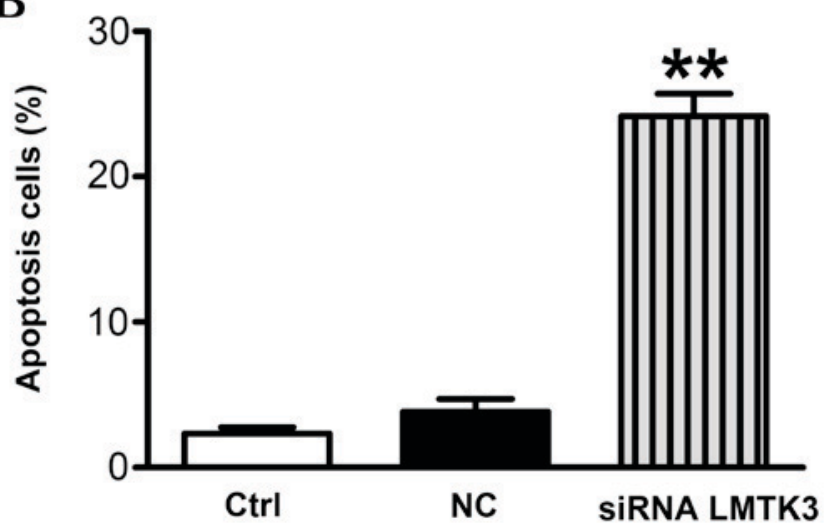

Figure 5. LMTK3 knockdown promoted apoptosis in SW579 cells. (A) Apoptotic cells were determined by TUNEL staining and visualized (magnification, x200). Green=apoptotic cells; blue=cell nucleus stained by DAPI. Representative images are presented. (B) Calculation of apoptotic cells. The results indicate that LMTK3 knockdown induced apoptosis in SW579 cells ( $\mathrm{n}=6$ independent experiments). Data are expressed as the mean \pm standard error of the mean. ${ }^{* *} \mathrm{P}<0.01$ vs. NC. Ctrl, control group; NC, negative control; siRNA, small interfering RNA; LMTK3, lemur tyrosine kinase-3; DAPI, 4',6-diamidino-2-phenylindole; TUNEL, terminal dUTP nick end labeling.

cells were stained using the TUNEL method. The number of apoptotic-positive cells was counted in a high-power field (magnification, $\mathrm{x} 200$ ). Representative images are presented in Fig. 5A. A notable increase in the number of apoptotic-positive SW579 cells was observed in the LMTK3 siRNA treatment group compared with the Ctrl group. However, the NC group displayed no significant differences (Fig. 5B).

\section{Discussion}

LMTK3 has been identified as a potential biomarker or prognostic factor in numerous types of cancer, including breast, gastric and colorectal cancer. In all of these, it has been shown to be increased in cancer cells compared with normal tissues $(1,27,28)$. In the present study, a critical association was also revealed between the serum LMTK3 level and thyroid cancer. It was identified, to the best of our knowledge for the first time, that the level of serum and tissue LMTK3 was markedly increased in patients with thyroid cancer (Figs. 1 and 2). This indicated that the serum LMTK3 level may be an important diagnostic and prognostic marker in thyroid cancer. The diagnostic and prognostic significance of the pre-operative serum LMTK3 level have been reported in numerous types of cancer. In colorectal cancer patients, the serum LMTK3 level was reported to be clearly higher compared with healthy volunteers, suggesting that serum LMTK3 could be a valuable biomarker for predicting the progression and prognosis of colorectal cancer (30). Similarly, in non-small cell lung cancer, the serum LMTK3 level was markedly elevated compared with a control group (31). Notably, the present study has identified that the protein and mRNA level of LMTK3 were significantly increased in patients with thyroid cancer. Therefore, it may be surmised that LMTK3 is involved in the pathological progression of thyroid cancer. The present results suggested that LMTK3 knockdown could dominantly inhibit proliferation, invasion and migration of SW579 cells (Figs. 3 and 4). In addition, the results gave a clear indication that suppression of LMTK3 could promote apoptosis in SW579 cells (Fig. 5). These findings not only helped to elucidate details of the mechanism of LMTK3 in regulating the proliferation, invasion and apoptosis in thyroid cancer cells, but also advanced the hypothesis that LMTK3 may serve as a novel therapeutic target for patients with thyroid cancer.

Hormone-related cancers, including breast, endometrial, ovarian and thyroid cancer, share carcinogenic mechanisms (15). Zhao et al (32) indicated that the exogenous delivery of miRNA to target LMTK3 could inhibit cell proliferation in the human breast cancer MCF-7 cell line. Recently, 
it has been demonstrated that LMTK3 co-localizes with ER in the nucleus, increasing ER transcription, stability and activity, which is closely associated with progression and disease outcome in breast cancer cells $(24,27)$. Notably, in the present study it was shown that the increased incidence of thyroid cancer is closely associated with dysregulation of LMTK3 in females (Table I). The results also demonstrated that the LMTK3 level was positively associated with the disease stage and pathological type (Table II). Taking into account the above results and the high level of ER receptor in thyroid cancer, it may be hypothesized that LMTK3 knockdown reduced proliferation, invasion and migration of thyroid cancer cells, partly by mediating ER activity. However, the underlying molecular mechanism governing how LMTK3 mediates ER activity remains to be explored.

In conclusion, the results of the present study demonstrated that the serum level of LMTK3 is associated with thyroid cancer and the disease stage, and thus LMTK3 may be a useful biomarker for the diagnosis and prognosis of thyroid cancer. In addition, LMTK3 knockdown could inhibit proliferation, migration and invasion of thyroid cancer cells. Therefore, LMTK3 may serve as a novel therapeutic target for patients with thyroid cancer. However, the exact mechanism of LMTK3 in thyroid cancer cells requires further investigation.

\section{References}

1. Ito Y, Nikiforov YE, Schlumberger M and Vigneri R: Increasing incidence of thyroid cancer: Controversies explored. Nat Rev Endocrinol 9: 178-184, 2013.

2. Kilfoy BA, Zheng T, Holford TR, Han X, Ward MH, Sjodin A, Zhang Y, Bai Y, Zhu C, Guo GL, et al: International patterns and trends in thyroid cancer incidence, 1973-2002. Cancer Causes Control 20: 525-531, 2009.

3. Pellegriti G, Frasca F, Regalbuto C, Squatrito S and Vigneri R: Worldwide increasing incidence of thyroid cancer: Update on epidemiology and risk factors. J Cancer Epidemiol 2013: 965212, 2013.

4. Hundahl SA, Fleming ID, Fremgen AM and Menck HR: A national cancer data base report on 53,856 cases of thyroid carcinoma treated in the U.S., 1985-1995 [see commetns]. Cancer 83: 2638-2648, 1998.

5. Xing M: Molecular pathogenesis and mechanisms of thyroid cancer. Nat Rev Cancer 13: 184-199, 2013.

6. Omur O and Baran Y: An update on molecular biology of thyroid cancers. Crit Rev Oncol Hematol 90: 233-252, 2014.

7. Huang IC, Chou FF, Liu RT, Tung SC, Chen JF, Kuo MC, Hsieh CJ and Wang PW: Long-term outcomes of distant metastasis from differentiated thyroid carcinoma. Clin Endocrino (Oxf) 76: 439-447, 2012.

8. Kebebew E, Weng J, Bauer J, Ranvier G, Clark OH, Duh QY, Shibru D, Bastian B and Griffin A: The prevalence and prognostic value of BRAF mutation in thyroid cancer. Ann Surg 246 466-471, 2007.

9. Shen WT, Ogawa L, Ruan D, Suh I, Duh QY and Clark OH: Central neck lymph node dissection for papillary thyroid cancer: The reliability of surgeon judgment in predicting which patients will benefit. Surgery 148: 398-403, 2010.

10. Baek SK, Jung KY, Kang SM, Kwon SY, Woo JS, Cho SH and Chung EJ: Clinical risk factors associated with cervical lymph node recurrence in papillary thyroid carcinoma. Thyroid 20: $147-152,2010$

11. Dorn R, Kopp J, Vogt H, Heidenreich P, Carroll RG and Gulec SA: Dosimetry-guided radioactive iodine treatment in patients with metastatic differentiated thyroid cancer: Largest safe dose using a risk-adapted approach. J Nucl Med 44: 451-456, 2003.
12. Wartofsky L, Sherman SI, Gopal J, Schlumberger M and Hay ID: The use of radioactive iodine in patients with papillary and follicular thyroid cancer. J Clin Endocrinol Metab 83: 4195-4203, 1998.

13. Bhaijee F and Nikiforov YE: Molecular analysis of thyroid tumors. Endocr Pathol 22: 126-133, 2011.

14. Nikiforov YE: Molecular analysis of thyroid tumors. Mod Pathol 24 (Suppl 2): S34-S43, 2011.

15. Henderson BE and Feigelson HS: Hormonal carcinogenesis. Carcinogenesis 21: 427-433, 2000.

16. Chen GG, Vlantis AC, Zeng Q and van Hasselt CA: Regulation of cell growth by estrogen signaling and potential targets in thyroid cancer. Curr Cancer Drug Targets 8: 367-377, 2008.

17. Rahbari R, Zhang L and Kebebew E: Thyroid cancer gender disparity. Future Oncol 6: 1771-1779, 2010.

18. Kansakar E, Chang YJ, Mehrabi M and Mittal V: Expression of estrogen receptor, progesterone receptor, and vascular endothelial growth factor-A in thyroid cancer. Am Surg 75: 785-789, 2009.

19. Lee HR, Kim TH and Choi KC: Functions and physiological roles of two types of estrogen receptors, ER $\alpha$ and ER $\beta$, identified by estrogen receptor knockout mouse. Lab Anim Res 28: 71-76, 2012.

20. Zeng Q, Chen GG, Vlantis AC and van Hasselt CA: Oestrogen mediates the growth of human thyroid carcinoma cells via an oestrogen receptor-ERK pathway. Cell Prolif 40: 921-935, 2007.

21. Inoue T, Kon T, Ohkura R, Yamakawa H, Ohara O, Yokota J and Sutoh K: BREK/LMTK2 is a myosin VI-binding protein involved in endosomal membrane trafficking. Genes Cells 13: 483-495, 2008.

22. Naik S, Dothager RS, Marasa J, Lewis CL and Piwnica-Worms D: Vascular endothelial growth factor receptor-1 is synthetic lethal to aberrant $\{$ beta\}-catenin activation in colon cancer. Clin Cancer Res 15: 7529-7537, 2009.

23. Tyner JW, Deininger MW, Loriaux MM, Chang BH, Gotlib JR, Willis SG, Erickson H, Kovacsovics T, O'Hare T, Heinrich MC and Druker BJ: RNAi screen for rapid therapeutic target identification in leukemia patients. Proc Natl Acad Sci USA 106: 8695-8700, 2009.

24. Giamas G, Filipović A, Jacob J, Messier W, Zhang H, Yang D, Zhang W, Shifa BA, Photiou A, Tralau-Stewart C, et al: Kinome screening for regulators of the estrogen receptor identifies LMTK3 as a new therapeutic target in breast cancer. Nat Med 17: 715-719, 2011.

25. Nikiforov YE: Molecular diagnostics of thyroid tumors. Arch Pathol Lab Med 135: 569-577, 2011.

26. Shi H, Li Q, Ji M, Wu J, Li Z, Zheng X, Xu B, Chen L, Li X, $\mathrm{Lu} \mathrm{C}$, et al: Lemur tyrosine kinase-3 is a significant prognostic marker for patients with colorectal cancer. Int J Clin Exp Pathol 7: 1101-1107, 2014.

27. Stebbing J, Filipovic A, Ellis IO, Green AR, D'Silva TR, Lenz HJ, Coombes RC, Wang T, Lee SC and Giamas G: LMTK3 expression in breast cancer: Association with tumor phenotype and clinical outcome. Breast Cancer Res Treat 132: 537-544, 2012.

28. Li Z, Wu J, Ji M, Shi L, Xu B, Jiang J and Wu C: Prognostic role of lemur tyrosine kinase 3 in postoperative gastric cancer. Mol Clin Oncol 2: 756-760, 2014

29. Fan Y, Chen M, Meng J, Yu L, Tu Y, Wan L, Fang K and Zhu W: Arsenic trioxide and resveratrol show synergistic anti-leukemia activity and neutralized cardiotoxicity. PLoS One 9: e105890, 2014.

30. Shi H, Wu J, Ji M, Zhou Q, Li Z, Zheng X, Xu B, Deng H, Zhao W, $\mathrm{Wu} \mathrm{C}$ and Jiang J: Serum lemur tyrosine kinase 3 expression in colorectal cancer patients predicts cancer progression and prognosis. Med Oncol 30: 754, 2013.

31. Xu Z, Qi X, Zhang X and Yu L: Preoperative serum LMTK3 as a novel biomarker in non-small cell lung cancer. Tumour Biol 35: 5007-5011, 2014

32. Zhao G, Guo J, Li D, Jia C, Yin W, Sun R, Lv Z and Cong X: MicroRNA-34a suppresses cell proliferation by targeting LMTK 3 in human breast cancer mcf-7 cell line. DNA Cell Biol 32: 699-707, 2013. 